\title{
Micro- and Nanotechnologies for Sensors
}

\author{
G. Urban, Albert-Ludwigs-Universität Freiburg ,Department of microsystems technologies, laboratory \\ for sensors, Georges Koehler Allee 103, Urban@imtek.de
}

\begin{abstract}
Recent progress in microsystems technologies has raised the expectation to get a comprehensive insight into metabolic events of patients. Such micro- and nanobiosensors in combination with appropriate micro fluidics enable the simultaneous description and moreover monitoring of gene, protein expression and metabolic states in a biosystem. In this paper some aspects of microfluidic devices for point of care applications and the use of nanomaterials for sensing purposes are addressed.
\end{abstract}

Key words: Microsensors, microfluidic devices, point of care, nanomaterials, nanosensors.

\section{Introduction}

At present, all intermediate acute testing of metabolic parameters are made using small point of care (POC) analysers which work with miniaturized sensor arrays.

To detect a variety of metabolic parameters precisely and selectively in a complex analyte matrix micro-and nanobiosensors have to be used.

\section{Microbiosensors}

Chiefly miniaturized glucose sensors faced an immense boom due to monitoring of diabetic patients at home allowing a more precise adjustment of insulin.

Such microbiosensors can be integrated to analyze different blood parameters at once and implemented in a microfluidics or onto a highly miniaturized device monitoring of body fluids can be done [1].

Additionally microfluidic and nanotechnological systems on chip exhibit the potential of cell based analysis especially pathogen detection on chip (fig.1).

Using special microfluidic structures called "phase guides" the defined filling and handling of biological samples on chip can be accomplished [2]. Enrichement, lysis, purification and isothermal amplification of cell specific oligonucleotides can be done on chip without moving parts and using only $5 \mu$ lanalyt volumes [3].

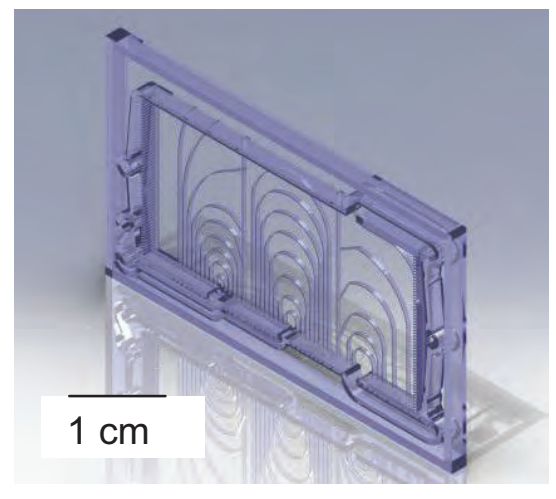

Fig. 1. Microfluidic chip for pathogen enrichment and detection

\section{Nanobiosensors}

The next step in the field of biosensors will be the use of nanotechnology: This was proven by the recent advances in next generation sequencing devices [4] as well as minimal invasive metabolic sensors [1].

Examples for the use of nanotransducers are cantilever based sensors which utilize a micromechanically produced cantilever in a similar manner as for production of AFM probes. The sensitivity can be tuned down to single molecule interaction analysis [5].

Recently the field of nanobiosensors emerged rapidly with different technologies to get insight into cellular metabolic events.

Also electrochemical sensing methods can be scaled down. Special attention was laid on carbon nanotubes modification of electrochemical working electrodes. A very 
exciting field is the use of carbon based nanostructures as new material for biosensing and biofuel cells.

Using such nanomodified electrodes high yield biofuel cells were accomplished which can be used for energy harvesting applications in biomedical applications [6].

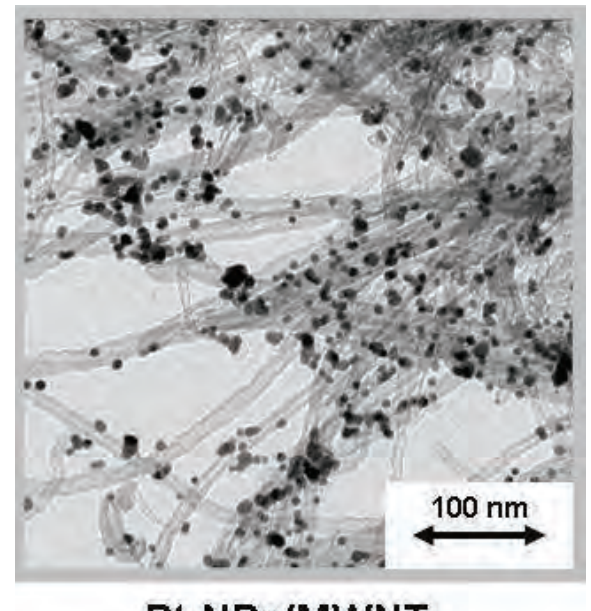

\section{Pt-NPs/MWNT}

Fig. 2. Micrograph of carbon nanotubes decorated with metallic nanoparticles for catalytic electrodes

\section{Theranostic devices}

The combination of analytical and therapeutic devices for implants the so called "theranostic" implants combining all such features will lead to the next generation of medical devices. The use of sensor nanosystems for implants is mandatory for monitoring biomarkers in blood and by using sophisticated biocompatible nanocoatings a better biostability and antimicrobial behavior can be realized. Using magnetron assisted plasma polymerization biocompatible nanofilms can be produced which are used in commercial available contact lenses [7].

\section{Resume}

Micro- and nanosystem based sensors are very promising candidates to see an improvement in bio- and chemosensing.

However, such tools are still in its infancy for biomedical applications but exhibit excellent potentials for the future.

\section{References}

[1] Moser I, Jobst G, Urban G, Biosensor Arrays for Simultaneous Measurement of Glucose, Lactate, Glutamate and Glutamine, Biosens Bioelectron, Band: 17, Nr. 4, 297 - 302 (2002)

[2] P. Vulto, G. Urban, Phaseguides: a paradigm shift in microfluidic priming and emptying, Lab on Chip, 11 1596-1602 (2011)

[3] Hakenberg S, Huegle M, Weidmann M, Hufert F, Dame G, Urban G, A Phaseguided Passive Batch Microfluidic Mixing Chamber for Isothermal Amplification, Lab on Chip 12, Nr 21, 4576 - 4580 (2012)

[4] Jonathan M Rothberg et. Al., An integrated semiconductor device enabling non-optical genome sequencing, Nature 475, 7356. 348352 (2011)

[5] Yin Tsung-I, Yunpeng Z, Horak J, Bakirci H, Liao $\mathrm{H}-\mathrm{H}$, Tsai $\mathrm{H}-\mathrm{H}$, Juang $\mathrm{Y}-\mathrm{Z}$, Urban $\mathrm{G}, \mathrm{A}$ Micro-cantilever Sensor Chip Based on Contact Angle Analysis for a Label-Free Troponin I Immunoassay, Lab on Chip 13, 834-842 (2013)

[6] Bunte C, Hussein L, Urban G, Performance of Non-Compartmentalized Enzymatic Biofuel Cell Based on Buckypaper Cathode and Ferrocene-Containing Redox Polymer Anode, J Power Sources Volume 247, 579586 (2013)

[7] Yasuda H, Ledernez L, Olcaytug F, Dame G, Bergmann M, Biocompatible Nanofilm Coating by Magneto-Luminous Polymerization of Methane , Prog Org Coat, 74, Nr 4, 667 - 678 (2012) 